\title{
Diagnóstico de la Gestión de Residuos Sólidos Urbanos en el Municipio de Mexicali, México: Retos para el Logro de una Planeación Sustentable
}

\author{
Crescencio L. Calva-Alejo ${ }^{(1)}$ y Rosa I. Rojas-Caldelas(2) \\ (1) Universidad Autónoma de Baja California. Facultad de Arquitectura y Diseño, Blvd. Benito Juárez S/N, \\ Col. Maestros Federales, C.P. 21280 Mexicali, Baja California-México (e-mail: Icalva@uabc.edu.mx). \\ (2) Universidad Autónoma de Baja California, Facultad de Arquitectura y Diseño, Blvd. Benito Juárez S/N, \\ Col. Maestros Federales, C.P. 21280 Mexicali, Baja California-México (e-mail: chicalirose@hotmail.com).
}

Recibido Oct. 1, 2013; Aceptado Dic. 10, 2013; Versión final recibida Feb. 11, 2014

\begin{abstract}
Resumen
Este trabajo tiene el propósito de evaluar la gestión municipal de Residuos Sólidos Urbanos en el municipio de Mexicali, México a través del marco de la sustentabilidad. Las áreas urbanas representan un foco de atención para las administraciones locales ya que representan espacios de importancia económica en el Producto Interno Bruto. Al mismo tiempo, en estas áreas se favorece la concentración de población y la contaminación del aire, agua y suelo en estas áreas. El análisis comprendió revisión documental, entrevistas semi-estructuradas y talleres participativos, agrupada en tres apartados: marco jurídiconormativo, buenas prácticas de gestión sustentable de residuos sólidos urbanos y diagnóstico municipal. El balance muestra avances en el marco jurídico con una débil instrumentación operativa y la necesidad de crear o reforzar el desarrollo de investigación, conformación de mercados, monitoreo, información y participación pública.
\end{abstract}

\section{Assessment of Municipal solid waste Management in the Municipality of Mexicali, Mexico: Challenges for Achieving Sustainable Planning}

\begin{abstract}
This work aims to evaluate the municipal management of urban solid wastes in the municipality of Mexicali, in Mexico through a sustainability framework. Urban areas are key issues for local governments since they are spaces whose production contributes significantly to the gross domestic product. At the same time, concentration of population in these areas and thereby increase of air, water and soil pollution are favored. The analysis included literature review, semi-structured interviews and participatory workshops, where information was grouped into three sections: legal and regulatory framework, best practices in sustainable management of urban solid wastes and municipal assessment. The overall balance shows advances in the legal aspects but regulations are poorly implemented. Also, it is needed to reinforce the development of research, market creation, monitoring, information and public participation.
\end{abstract}

Keywords: urban planning, sustainable management, urban solid wastes, municipal environmental management 


\section{INTRODUCCIÓN}

Actualmente, la mayoría de la población mundial se concentra en las zonas urbanas, presentándose retos cada vez mayores para resolver problemas urbano-ambientales y de gobernanza en las ciudades producto de una acelerada urbanización iniciada a partir de mediados del siglo XX, que formó ciudades que no se prepararon para las demandas de sus habitantes y su crecimiento adquirió un carácter desordenado (Jordán, 2003; SEDESOL, 2011; Aponte, 2007). De acuerdo a las proyecciones de crecimiento de población a nivel mundial para 2011 se contaba con 7 mil millones de habitantes, tendencia que seguirá en aumento, donde algunos escenarios indican que los 9 mil millones se alcanzarán para el 2043, imponiendo cada vez mayores presiones sobre los recursos naturales y su respectiva disposición (UNEP, 2012).

México es un país eminentemente urbano. De acuerdo al censo de población 2010 , se indica que el $72 \%$ de la población del país vive en 383 ciudades con más de 15,000 habitantes. Las proyecciones actuales del fenómeno urbano en México, estiman que la población llegará a 121 millones de personas en los próximos 18 años, que habitarán en ciudades de más de un millón de habitantes en 2030 (SEDESOL, 2011). Dicho crecimiento y concentración demandará diversos insumos para su sostenimiento como agua, energía y materiales, así como la adecuada disposición de desechos vertidos al agua, aire y suelo, donde el vertido inadecuado de residuos sólidos altera la calidad de estos tres recursos así como su impacto en la salud humana y de los ecosistemas.

El tema de los residuos sólidos urbanos constituye una de las mayores preocupaciones de las sociedades contemporáneas y un desafío mundial para la gestión pública (AIDIS, 2006). Su creciente aumento está relacionado directamente con el crecimiento demográfico, la concentración en áreas urbanas o metropolitanas, los procesos productivos que no han logrado cumplir con la normatividad ambiental y un modelo económico que tiene efectos negativos en los hábitos de consumo de la población (De Valle, 2005; Zaman y Lehmann, 2011; Zaman, 2014; Vij, 2012). Este problema se expresa como tendencia mundial en la generación de residuos sólidos urbanos, donde los mayores niveles corresponden a los países con ingresos económicos altos (Kharvel, 2012), tal es el caso de la región de América del Norte, conformada por Estados Unidos, Canadá y México (UNEP, 2004).

En México, la transición de lo rural a lo urbano, modificó los patrones de consumo de una sociedad que producía mayormente residuos orgánicos, a una que produce principalmente residuos inorgánicos derivados de los patrones de consumo típicos de sociedades industriales urbanas (SEDESOL, 2011). Tan solo en el 2010, el país generó diariamente 109,750 toneladas de residuos sólidos, de éstos el $64 \%$ se depositaron en rellenos sanitarios, el $9 \%$ en rellenos de tierra controlados, y el restante $27 \%$ se dispuso en sitios no controlados. A pesar de que menos de la tercera parte de los residuos sólidos terminan en sitios no controlados, esta práctica genera graves problemas ambientales que afectan la salud y la seguridad de los residentes de las localidades circundantes (INEGI, 2010). Se ha caracterizado la generación per cápita promedio diario en $0.9 \mathrm{~kg}$, considerando las zonas rurales con $0.4 \mathrm{~kg}$ y en las zonas metropolitanas de 1.5 kg. (INE, 2010).

La gestión de los residuos sólidos urbanos en la mayoría de los países en desarrollo recae en las autoridades locales, como en China, Turquía, India, Etiopía, Uganda, Grecia y España entre otros (Chen et al., 2010; Kanat, 2010; Lohri et al., 2014; Okot-Okumo y Nyenje, 2011; Papachristou et al., 2009; Zamorano et al., 2009), donde el caso de México no escapa a esta condición, la "Constitución Política de los Estados Unidos Mexicanos" señala que es responsabilidad de las autoridades municipales su recolección y manejo (SEDESOL, 2005). A continuación se presenta la evolución que ha tenido este tema en diferentes periodos de cambios de la legislación, producto de la visión que se tenía en un contexto histórico determinado sobre los residuos sólidos y respecto a la falta de capacidades de gestión para dotar la infraestructura y agenciarse de recursos financieros por parte de los Ayuntamientos para atender este problema (Gutiérrez, 2006; GTZ, 2003).

\section{GESTIÓN DE RESIDUOS SÓLIDOS EN MÉXICO}

La gestión de residuos sólidos en México pasa por tres momentos en su historia: inicia en 1964 bajo un enfoque predominante de regulación sanitaria, posteriormente en 1988 a partir de la creación de la legislación ambiental nacional se da un paso hacia el manejo básico de los residuos, el último cambio en 2003 obedece a la creación de la Ley General para la Prevención y Gestión Integral de los Residuos (DOF, 2003), enfoque que va más allá de las propuestas anteriores, al incorporar algunos aspectos del manejo sustentable de los residuos sólidos urbanos (Zaman y Lehmann, 2014; Ezeah y Roberts, 2014; Menikpura et al., 2013; Lohri et al., 2013; Dálisa et al., 2012; Marshall y Farahbakhsh, 2013; Pires et al., 2011), enfoques que se muestran en la Tabla 1. 
Tabla 1: Diferencias entre los enfoques establecidos en la legislación mexicana: regulación sanitaria, manejo básico y gestión integral de residuos sólidos urbanos, respecto del marco conceptual de la gestión sustentable de residuos sólidos urbanos (Elaboración propia)

\begin{tabular}{|c|c|c|c|}
\hline $\begin{array}{l}\text { Marco conceptual: gestión } \\
\text { sustentable de rsu }\end{array}$ & $\begin{array}{l}\text { Regulación sanitaria de } \\
\text { residuos } 1964-1987\end{array}$ & $\begin{array}{l}\text { Manejo básico de residuos } \\
1988-2002\end{array}$ & $\begin{array}{l}\text { Gestión integral de RSU } \\
2003-2012\end{array}$ \\
\hline $\begin{array}{l}\text { Producción de bienes y } \\
\text { ambiente }\end{array}$ & $\begin{array}{l}\text { Ambiente y desarrollo } \\
\text { separados }\end{array}$ & $\begin{array}{l}\text { Se involucra el tema } \\
\text { ambiental en la producción } \\
\text { de bienes y servicios. }\end{array}$ & $\begin{array}{l}\text { Se involucra la } \\
\text { responsabilidad ambiental }\end{array}$ \\
\hline Metabolismo urbano & Metabolismo lineal & Metabolismo circular & Metabolismo circular \\
\hline $\begin{array}{l}\text { Recuperación de } \\
\text { materiales }\end{array}$ & Recuperación mínima & $\begin{array}{l}\text { Diversidad de } \\
\text { subproductos recuperados }\end{array}$ & $\begin{array}{l}\text { Destaca el manejo } \\
\text { jerárquico de residuos a } \\
\text { través de la reducción, } \\
\text { reutilización y reciclaje }\end{array}$ \\
\hline Infraestructura & $\begin{array}{l}\text { Recursos físicos y } \\
\text { humanos escasos }\end{array}$ & $\begin{array}{l}\text { Recursos escasos, con } \\
\text { implementaciones para la } \\
\text { recuperación }\end{array}$ & $\begin{array}{l}\text { Recursos escasos, con } \\
\text { mejoras en el acopio de } \\
\text { materiales }\end{array}$ \\
\hline Marco jurídico & $\begin{array}{l}\text { Marco legal general para } \\
\text { una gestión sanitaria }\end{array}$ & $\begin{array}{l}\text { Marco legal ambiental para } \\
\text { una gestión operativa }\end{array}$ & $\begin{array}{l}\text { Marco legal sectorial para } \\
\text { una gestión operativa }\end{array}$ \\
\hline Políticas públicas & $\begin{array}{l}\text { Acciones operativas de } \\
\text { corto plazo }\end{array}$ & $\begin{array}{l}\text { Políticas públicas } \\
\text { operativas y de corto plazo }\end{array}$ & $\begin{array}{l}\text { Políticas públicas } \\
\text { operativas de prevención } \\
\text { de la contaminación y de } \\
\text { corto plazo }\end{array}$ \\
\hline Innovación e investigación & $\begin{array}{l}\text { La investigación es mínima } \\
\text { y orientada a la salud }\end{array}$ & $\begin{array}{l}\text { Elaboración de estudios } \\
\text { técnicos, de acuerdo con } \\
\text { las normas oficiales }\end{array}$ & $\begin{array}{l}\text { Elaboración de estudios } \\
\text { técnicos, y auditorías } \\
\text { ambientales }\end{array}$ \\
\hline $\begin{array}{l}\text { Educación } \\
\text { y capacitación }\end{array}$ & $\begin{array}{l}\text { Ejes educativos sin } \\
\text { contenidos ambientales y } \\
\text { capacitación mínima }\end{array}$ & $\begin{array}{l}\text { Actividades de educación } \\
\text { ambiental formal e } \\
\text { informal. Capacitación } \\
\text { incipiente }\end{array}$ & $\begin{array}{l}\text { Se extienden los } \\
\text { contenidos en los ejes } \\
\text { educativos básicos. } \\
\text { Capacitación muy } \\
\text { localizada. }\end{array}$ \\
\hline Mercados & Mercado informal & $\begin{array}{l}\text { Se construyen circuitos de } \\
\text { comercio con personas } \\
\text { que recogen residuos y los } \\
\text { venden (pepenadores) }\end{array}$ & $\begin{array}{l}\text { Se desarrolla el mercado } \\
\text { de subproductos sin } \\
\text { esquemas de } \\
\text { financiamiento. }\end{array}$ \\
\hline Financiamiento & $\begin{array}{l}\text { Partidas para los servicios } \\
\text { públicos municipales }\end{array}$ & $\begin{array}{l}\text { Partidas para servicios } \\
\text { públicos municipales, que } \\
\text { resultan insuficientes para } \\
\text { las demandas }\end{array}$ & $\begin{array}{l}\text { Existe el cobro por la } \\
\text { prestación del servicio de } \\
\text { recolección }\end{array}$ \\
\hline Participación social & $\begin{array}{l}\text { Toma de decisiones sin } \\
\text { involucrar a la ciudadanía. }\end{array}$ & $\begin{array}{l}\text { La sociedad civil } \\
\text { organizada participa, pero } \\
\text { marginalmente }\end{array}$ & $\begin{array}{l}\text { Promoción de la } \\
\text { participación de todos los } \\
\text { sectores de la sociedad }\end{array}$ \\
\hline Seguimiento y evaluación & $\begin{array}{l}\text { No se describe ningún tipo } \\
\text { de evaluación de tareas }\end{array}$ & $\begin{array}{l}\text { Se lleva seguimiento de } \\
\text { programas instrumentados, } \\
\text { pero su efectividad es } \\
\text { mínima. }\end{array}$ & $\begin{array}{l}\text { Se establece la integración } \\
\text { de sistemas de evaluación } \\
\text { de las políticas de } \\
\text { prevención de la } \\
\text { contaminación }\end{array}$ \\
\hline
\end{tabular}

Regulación sanitaria: este enfoque consideraba a los residuos sólidos como un problema de saneamiento urbano que estaba a cargo de las autoridades de salubridad pública, su operación se concretaba a la recolección y alejamiento de los residuos de los centros urbanos para su disposición en las zonas rurales con el manejo tradicional de la quema. El periodo de regulación sanitaria comprende desde la aparición de las primeras reglas federales de operación por parte de la Secretaría de Salubridad y Asistencia (SSA) en 1964, hasta la asignación de funciones a los servicios públicos en los municipios en 1985. Enfoque que se modifica posteriormente con la publicación de la Ley General del Equilibrio Ecológico y Protección al Ambiente en 1988 (DOF, 1988), al establecerse las normas oficiales mexicanas para los sitios de disposición final. 
Manejo Básico: este enfoque asumió no solo una política operativa de recolección y disposición final, sino también tareas de prevención de la contaminación por RSU. Con la publicación de la Ley del Equilibrio Ecológico y Protección al Ambiente, en 1988, se construyó la primera tipología de residuos y se distribuyeron competencias para los tres órdenes del gobierno, ratificando la formulación de la política ambiental, la prestación del servicio de recolección y manejo de RSU, a los municipios. Así también, aparece una Norma Oficial Mexicana específica en 1996 para los sitios de disposición final, y se acentúa la construcción de infraestructura a cargo del gobierno federal, al manifestarse las limitaciones presupuestales que tenían los ayuntamientos para construir este tipo de infraestructura. La vigencia de este enfoque se extiende hasta antes de la publicación de la primera ley sectorial federal (Gil, 2009).

Gestión integral de Residuos Sólidos Urbanos: este enfoque presenta una visión comprensiva a través de la publicación de la Ley General para la Prevención y Gestión Integral de los Residuos (DOF, 2003). Cuenta con una categorización en tres grupos de residuos: residuos sólidos urbanos (RSU) de competencia municipal, residuos de manejo especial (RME) de competencia estatal y residuos peligrosos (RP) de competencia federal. Bajo esta categorización los gobiernos locales asumen la responsabilidad de la gestión municipal, gestión que exige la coordinación y concurrencia con los otros niveles de gobierno para el control de la contaminación bajo la suscripción de convenios. Así la gestión integral considera, atender el sistema de manejo de residuos que incluye la generación, almacenamiento, barrido, recolección, traslado, tratamiento, aprovechamiento de materiales y disposición final, al igual que comprender acciones normativas para la expedición de reglamentos de limpieza, estímulos para la reducción de la basura, la promoción de centros de acopio, gestión de recursos y apoyos, capacitación, así como evaluaciones de impacto al medio ambiente natural y social.

Con la publicación de esta primera ley federal sectorial, el enfoque que se mantiene vigente, es el establecido en el Programa Nacional para la Prevención y Gestión Integral de los Residuos 2009-2012 (SEMARNAT, 2009), que asigna nuevos principios de gestión al municipio para atender a los RSU, a través de una política basada en la promoción de cambios en los modelos de producción y consumo, minimización en la generación, su valorización y disposición final apropiada, para contribuir al desarrollo sustentable del país.

Este marco define entonces el enfoque, competencias y tipo de residuos que debe atender la administración municipal acotándolo a los RSU, definidos como aquellos generados en las casas habitación, que resultan de la eliminación de los materiales que utilizan en sus actividades domésticas, de los productos que consumen y de sus envases, embalajes o empaques; los residuos que provienen de cualquier otra actividad dentro de establecimientos o en la vía pública que genere residuos con características domiciliarias y los resultantes de la limpieza de las vías y lugares públicos, siempre que no sean considerados por esta Ley, como residuos de otra índole.

\section{METODOLOGÍAS PARA LA PLANEACIÓN SUSTENTABLE DE RESIDUOS SÓLIDOS}

Este apartado basa su desarrollo en la revisión de trabajos teóricos y metodológicos, así como de buenas prácticas implementadas en varios países para la gestión integral y/o sustentable de residuos sólidos en el ámbito municipal, materiales que han aportado por un lado, los conceptos a ser incluidos en el diseño de la metodología de diagnóstico del estudio de caso de Mexicali, y por otro, las metodologías de planeación, las limitaciones y retos que enfrentan en la práctica las administraciones municipales en materia de RSU (CYMA, 2008; Consorcio Cívico, 2010; ICLEI, 2005; UNEP, 2009; AUMA, 2006; Morinville, 2010).

Las propuestas en general están construidas a partir del enfoque de metabolismo circular para residuos sólidos que integra formas compatibles de producción y consumo que consideran los flujos de materia y energía en el sistema, que remite al aprovechamiento de los residuos vía la reducción en el consumo, la reutilización y reciclaje (Dálisa et al., 2012; Marshall y Farahbakhsh, 2013; Pires et al., 2011; Seadon, 2010). Además de contemplar la restauración ambiental; el establecimiento de nuevas formas administrativas y normativas; la reingeniería de políticas públicas; la incorporación de la innovación tecnológica y el desarrollo de la investigación, el fomento de ciclos educativos y de capacitación, la formación de circuitos de mercados, conformación de clústeres, la participación de la iniciativa privada, la gestión y fomento de finanzas al sector, fondos e inversión; la participación pública; y la ejecución de un seguimiento y evaluación (ICLEI, 2005, AUMA, 2006; Centro Complutense de Estudios e Información Medioambiental, 2009). Estos elementos sirvieron de base para analizar las formas de gestión que se han tenido en México y del diagnóstico que se llevó a cabo en su aplicación al caso del municipio de Mexicali. La figura 1 muestra los elementos que forman el marco para la planeación de los Residuos Sólidos Urbanos desde el enfoque de la sustentabilidad, materia central de este trabajo. 


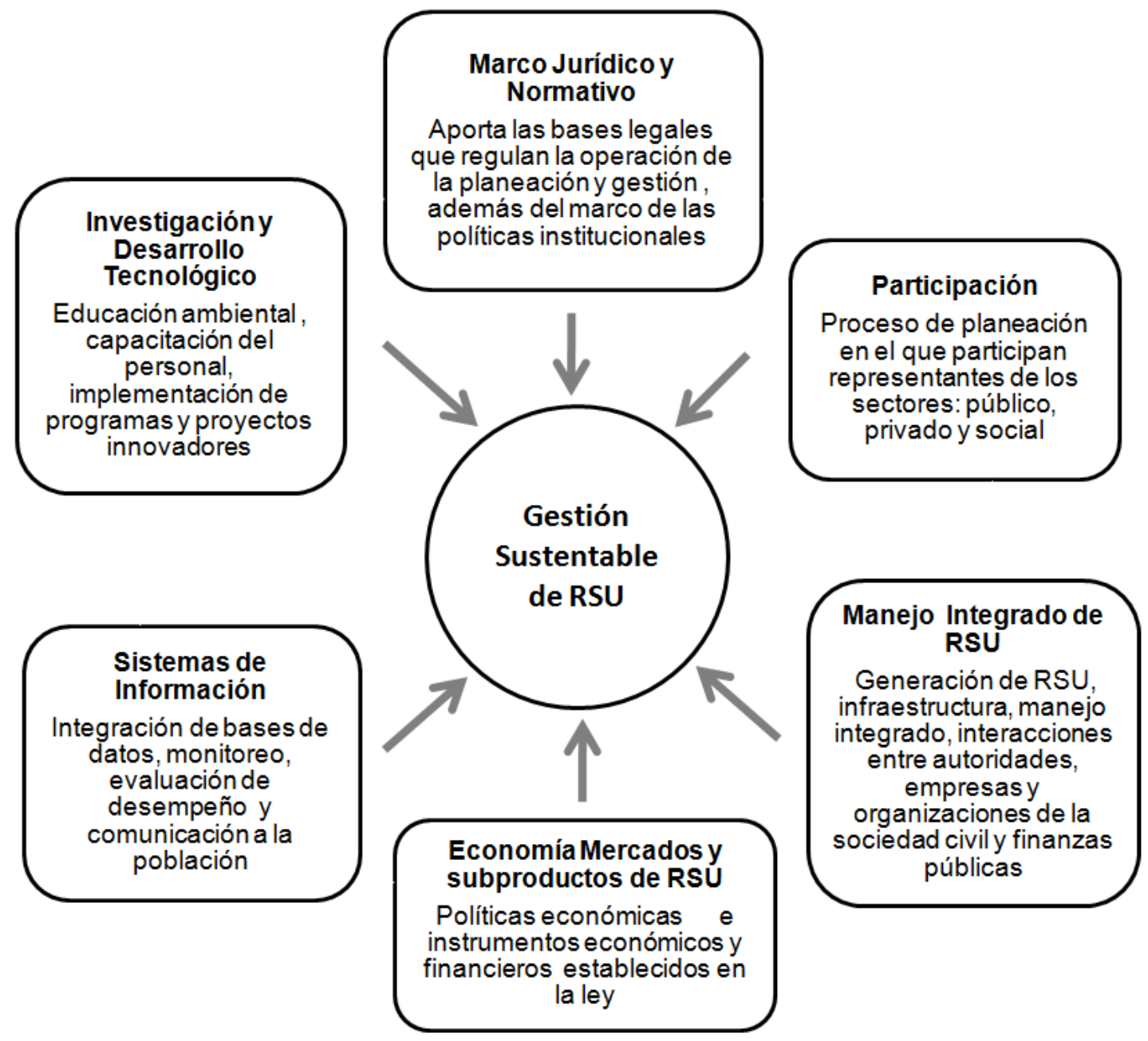

Fig. 1: Esquema muestra los elementos que conforman el marco para la planeación de los Residuos Sólidos Urbanos desde el enfoque de la sustentabilidad

De acuerdo al esquema propuesto (Fig.1), se realizó el diagnóstico del caso de estudio sobre la gestión de los RSU en el municipio de Mexicali a través de los siguientes rubros: Marco Jurídico y Normativo, describe las competencias y atribuciones de los órdenes del gobierno en el sector, referenciando la estructura normativa federal, estatal y municipal; Marco Institucional, presenta las políticas y acciones concernientes a planes y programas que lleva a cabo el Ayuntamiento de Mexicali, a través de las dependencias relacionadas con el tema; Participación, integra las diferentes formas de participación en actividades de planeación y gestión que realizan los actores, así como los derechos que tienen de acceso a la información y mecanismos de difusión de acciones de seguimiento y evaluación del desempeño que realiza el Ayuntamiento. Actores, grupos involucrados en la gestión de los residuos, este análisis facilita la identificación de perfiles y opiniones diversas sobre el sistema de gestión en el que participan: responsables, grupos y organizaciones del sector público, privado y social ligados al tema; Manejo Integrado de RSU, proporciona la información básica sobre generación, transferencia y disposición; Infraestructura, reporta los recursos materiales e instalaciones que se cuentan para llevar a cabo el trabajo de manejo de los residuos por parte del Ayuntamiento; Interacciones, muestra las acciones de concurrencia que tiene el Ayuntamiento con los otros niveles de gobierno, los residuos de manejo especial y peligrosos; Finanzas, proporciona la información presupuestaria para la operación del servicio por parte del Ayuntamiento; Economía, considera las oportunidades de nuevos nichos de mercado para el aprovechamiento de los subproductos de los residuos sólidos y de los instrumentos económicos y financieros a que pueden recurrir las empresas; Sistemas de Información, crear las bases de datos asociadas a la planeación y gestión de los residuos sólidos para una mejor toma de decisiones y de acciones de evaluación y seguimiento de su desempeño y comunicación a la sociedad; Investigación y Desarrollo, incorpora las contribuciones científicas y tecnológicas orientadas al manejo sustentable de los residuos sólidos, al igual considera la educación ambiental como base de los cambios culturales, como la importancia que tiene la difusión y 
comunicación de acciones a la sociedad y la retroalimentación que hagan a la gestión y; Educación y capacitación, provee educación ambiental no formal, capacita a su personal e implementa acciones de innovación tecnológica en el manejo de RSU.

\section{DIAGNÓSTICO DE LOS RSU EN EL MUNICIPIO DE MEXICALI}

El trabajo que enseguida se presenta estuvo basado en tres tipos de fuentes: Documentales que integraron la revisión de leyes, reglamentos, normas, anuarios estadísticos, informes de gobierno, estudios específicos realizados sobre residuos sólidos, planes de desarrollo que contenían las políticas públicas (federal, estatal, municipal y para la ciudad) y buenas prácticas desarrolladas en otros países. Entrevistas semiestructuradas a funcionarios de la administración municipal relacionados con la planeación urbana, la protección ambiental y la prestación del servicio de recolección de RSU. Igualmente se hizo con el responsable de la empresa concesionada del servicio de transferencia y disposición final, el representante del sindicato de trabajadores de la pepena y de organizaciones sociales que promueven la educación ambiental y el reciclado de subproductos. Talleres Participativos, se realizaron tres que cubrieron los diferentes sectores del municipio, donde se aplicó un cuestionario estructurado a representantes de autoridades, empresarios, asociaciones profesionales, organizaciones de la sociedad civil y representantes académicos quienes aportaron su punto de vista y jerarquización de los problemas ambientales y en específico de los RSU. A partir del esquema mostrado en la fig. 1 se desarrolla el diagnóstico de RSU en el caso del municipio de Mexicali.

Marco Jurídico. En un principio, en Mexicali, las acciones de saneamiento se basaban en las reglas federales de la Secretaría de Salubridad y Asistencia, en la Ley Federal para Prevenir y Controlar la Contaminación Ambiental (Gil, 2009) y la Ley Federal de Protección al Ambiente (Gil, 1982), hasta lograr la asignación constitucional de los servicios públicos en 1985. Posteriormente la Ley General del Equilibrio Ecológico y Protección al Ambiente (LGEEPA) 1988 impulsa la política ambiental municipal, ratificando la atención de los RSU. La Ley General para la Prevención y Gestión Integral de los Residuos (LGPGIR) (DOF, 2003), incorpora una visión de mejora técnica y normativa de los procesos en el manejo de los residuos. Además de la observancia al marco federal, se ha avanzado en el orden estatal con la Ley de Protección al Ambiente y la Ley de Prevención y Gestión Integral de Residuos, y en el municipal, con el Reglamento para la Preservación del Aseo Público. Aún así, la gestión no ejecuta acciones con ese marco legal sectorial, manteniendo acciones operativas de recolección y disposición, desde los servicios públicos.

Marco Normativo Institucional. En el nivel local, al describirse un limitado desarrollo institucional del Municipio para atender la responsabilidad ambiental, el orden federal ejecutó acciones desde los primeros programas de atención a los RSU. Desde el Ayuntamiento, la Dirección de Servicios Públicos, es la responsable de la prestación del servicio de recolección, transporte a la estación de transferencia y posteriormente el transporte al sitio de disposición final. En el Valle de Mexicali, se delegan estas acciones a la Dirección de Desarrollo Rural y Delegaciones. Por su parte, la Dirección de Ecología revisa la normativa, con algunas acciones de promoción para la prevención de la contaminación y de educación ambiental y el Instituto Municipal de Planeación, ha localizado sitios de disposición final clandestina en el Valle, pero no genera ninguna tarea de planeación sobre el tema. En el organigrama del Ayuntamiento, no se cuenta con ninguna área específica para la atención de los RSU y se presenta escasa interacción con otros departamentos del Ayuntamiento.

Políticas públicas. Las primeras políticas públicas federales para resolver el problema de los RSU, estaban encaminadas primordialmente a las tareas de aseo urbano. Posteriormente con la Secretaria de Desarrollo Urbano y Ecología, se lleva la primera promoción de una política de educación ambiental. Con la publicación de la LGEEPA (DOF, 1988), se distribuyeron competencias para los tres órdenes del gobierno, ratificando la formulación de la política ambiental municipal y la prestación del servicio de recolección y manejo de RSU. Con el enfoque integral se avanzó en proponer la generación de políticas sectoriales que se plasmaron en el Plan Nacional de Desarrollo 2007-2012 y el Programa Nacional para la Prevención y Gestión Integral de los Residuos 2009-2012 (SEMARNAT, 2009). En el ámbito local, el Programa de Desarrollo Urbano de Centro de Población de Mexicali 2025, incluye a la gestión de los residuos sólidos urbanos como una línea de política ambiental, pero la ejecución de políticas públicas municipales, se sigue basando en tareas de recolección y disposición de RSU.

Participación. El enfoque integral asume la promoción de la participación de todos los sectores de la sociedad en la prevención de la generación, la valorización y gestión integral de residuos. En Mexicali, el proceso de gestión municipal en el tema de los RSU, no contempla mecanismos de participación social en la toma de decisiones. La participación social está encaminada a la promoción de jornadas de limpieza, con el apoyo de asociaciones civiles como Ecovida. A.C, y Mexicali te Queremos Limpio A.C. 
Actores. En un principio, la responsabilidad del manejo municipal de RSU estaba ejecutada únicamente por los funcionarios de la oficina de servicios públicos municipales, posteriormente al entrar en vigor las políticas neoliberales a finales de los ochentas, se implementan esquemas compartidos de gestión de los RSU entre sector público, privado y social, modalidad que hasta la fecha es vigente, de ahí que todos sean actores que desempeñan funciones diferentes en la gestión. En la actualidad, los actores involucrados en el sistema de gestión municipal, son el Director de Servicios Públicos Municipales a cargo de las acciones operativas de recolección, transporte y disposición, en la ciudad de Mexicali; el Director de Desarrollo Rural y Delegaciones (sectorización administrativa municipal), con las mismas acciones en el Valle; el Director del Instituto Municipal de Planeación, con trabajo de localización de sitios de disposición clandestina; la Directora de Ecología, en la revisión de planes de educación ambiental; y el Comité de Planeación para el Desarrollo Municipal de Mexicali (COPLADEMM), que funciona como consejo consultivo para la formulación, seguimiento y evaluación de las políticas y programas ambientales municipales. Comité integrado por los diferentes actores: funcionarios de los diferentes niveles de gobierno, representantes de las cámaras comerciales e industriales, empresas, instituciones académicas y de investigación, asociaciones profesionales y grupos de la sociedad civil organizada, aunque su participación es informativa y consultiva, donde los compromisos recaen únicamente en el sector gubernamental.

Gobernabilidad. Desde el enfoque integral la LGPGIR (2003) se recomienda a las autoridades del Municipio, adecuar los marcos legales y mejorar los procesos de manejo de residuos para la utilidad pública y el interés social. En Mexicali, el sistema de gestión para los RSU, es interrumpido con los procesos de transición política cada tres años, dejando compromisos de orden administrativo con el servicio concesionado (PASA. S.A de C.V) y el sindicato de trabajadores de la pepena (Unión de Bote y Chatarra A.C). Lo que ha llevado a un estado de ineficacia para manejar adecuadamente los RSU y la generación de una percepción de ingobernabilidad.

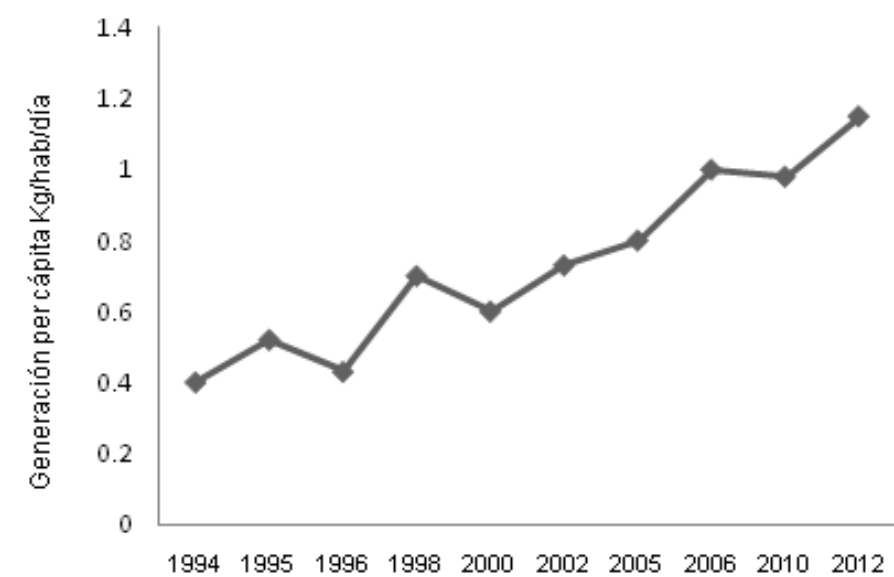

Fig. 2: Distintos datos y proyecciones anuales de generación de RSU para la ciudad de Mexicali (Datos de Cortinas (1994), Ojeda y Silva (1996), Ojeda, 1998, Grupo Técnico del Inventario Mexicali (1999), INE (2010) y XVIII Ayuntamiento de Mexicali (2007).

Generación de RSU. La información disponible sobre los volúmenes de generación de los RSU para la ciudad de Mexicali, es limitada y dispersa. Algunos datos sobre este aspecto refieren a que la generación per cápita en 1994 alcanzaba 0.433 Kg (Cortinas, 1994), posteriormente, los estudios realizados por Camacho-Gaxiola (1995), registraron $0.489 \mathrm{Kg}$; así también, Ojeda y Silva, (1996), puntualizaron un volumen de generación de $0.464 \mathrm{Kg}$; en el mismo nivel, el Inventario de Emisiones de Mexicali (Grupo Técnico del Inventario Mexicali, 1999), registró $0.6 \mathrm{Kg}$ por persona/día. Al respecto, se incorporaron distintas proyecciones de generación realizadas por el Instituto Nacional de Ecología, para 1997, $0.677 \mathrm{Kg}$. (INE, 2010), para el año 2000, $0.713 \mathrm{Kg}$. (INE, 2010), para el 2005, $0.781 \mathrm{Kg}$. (INE, 2010), y para el 2010, $0.97 \mathrm{Kg}$. (INE, 2010). Otro dato para el año 2005, se obtuvo del documento del Programa de Desarrollo Urbano del Centro de Población de Mexicali 2025 (XVIII Ayuntamiento de Mexicali, 2007), que promediaba $0.99 \mathrm{Kg}$ por persona al día. Por último, se incluyó la estimación del promedio de generación per cápita de $1.1 \mathrm{Kg}$. Aportada por la Dirección de Servicios Públicos Municipales del XX Ayuntamiento de Mexicali (2012) (Ver fig.2). El valle de Mexicali representa la zona rural del municipio, sin embargo su estilo de vida, patrones de consumo y generación de residuos, cada vez son más parecidos a los de la ciudad de Mexicali. De esta manera, los datos actuales de generación de RSU proporcionados por la Dirección de Desarrollo 
Rural y Delegaciones oscila alrededor de las cien mil toneladas anuales, con un promedio diario de 273 toneladas. Lo que representa la tendencia a un incremento en la generación por habitante, con el impacto asociado y su potenciación con las prácticas del modelo de gestión vigente.

Manejo integrado de RSU. Con base en el Taller participativo para la Elaboración de la Agenda Ambiental Metropolitana (SPA, 2011), el tema de los RSU se posicionó en el primer lugar de atención antes que cultura ambiental, contaminación del aire, agua, áreas verdes, contaminación física: lumínica, olores, sonora y electromagnética. En este tenor, los participantes demostraron conocimiento en general del manejo de los residuos, en la ciudad o localidades del Valle, en cuanto a la cobertura del servicio de recolección, la frecuencia de recolección domiciliaria, el pago por el servicio, la existencia de un relleno sanitario cercano y la recepción de información por parte de las autoridades; así como sobre las formas alternas de manejo de residuos en el caso que no se contara con este servicio municipal. En cuanto a las alternativas de disposición de residuos peligrosos, en la ciudad de Mexicali y el Valle, son escasos los trabajos de investigación en el tema, pero de acuerdo a Favela (2009), una gran fracción de los residuos sólidos urbanos que se generan puede contener un potencial de peligrosidad y se incorporan a los sitios de disposición o en gran medida se disponen clandestinamente, por lo que en este Taller, se consideró importante conocer los diferentes tipos de residuos peligrosos desechados a nivel doméstico. También se subrayó por los asistentes, que debería de haber mayor comunicación, corresponsabilidad y coordinación entre las prácticas de manejo de los residuos sólidos desarrolladas por los habitantes y la prestación del servicio de recolección municipal. En lo que respecta a la recuperación de materiales, los residentes manifestaron su colaboración en esta actividad de manera informal, donde destaca en primer lugar la recuperación de papel seguida por plásticos, metales, vidrio y aceites usados. Situación que expresa la importancia económica que tienen estos residuos para las empresas que participan en el mercado de reciclado. Igualmente se reporta esta actividad para residuos provenientes de comercios y servicios (Fierro, 2010; Garduño, 2012 y Armijo, 2006). En lo que toca a la infraestructura para el manejo de los residuos sólidos urbanos, el municipio cuenta el personal y transporte para la recolección domiciliaria, dos estaciones de transferencia, una en la periferia de la ciudad (Xochimilco) y otra en el valle de Mexicali (Ejido Delta) y el relleno sanitario ubicado en el Ejido Hipólito Rentería que cuenta con una vida útil al 2020, situación que demandó la ampliación de capacidad en un nuevo relleno sanitario en 2010. Los problemas operativos según autoridades y residentes se concretan en la falta de recursos económicos para solventar el costo de gasolina para dar el servicio de recolección dos veces a la semana y proporcionar el mantenimiento de las unidades.

Interacciones. En un inicio, el Ayuntamiento atendió el manejo de los RSU, sin construir concurrencias con otros niveles de gobierno y una interacción escasa con la iniciativa privada. Con el enfoque integral se hizo patente una concurrencia necesaria y la promoción de una interacción efectiva con la iniciativa privada. En Mexicali, la gestión se ha venido desarrollando con la concesión a empresas particulares. Actualmente PASA. S.A de C.V es la empresa que abarca, la recolección, transferencia, transporte y disposición final. Por su parte Winco y Rimsa prestan el servicio de recolección de residuos biológico-infecciosos a nivel municipal. Sobre las acciones de coordinación y concurrencia, con otros niveles de gobierno para atender otros tipos de residuos (manejo especial y los peligrosos), de acuerdo a la Dirección de Servicios Públicos, se mantiene una comunicación continua con la Secretaria de Protección al Ambiente del Estado de Baja California. En este sentido, no se especifican acciones, programas y proyectos ni se cuenta con ningún registro de contaminantes asociados a la actividad agrícola del Valle de Mexicali, ni con un padrón de empresas que presten servicios asociados a la gestión de residuos.

Financiamiento. El recurso para la prestación del servicio de recolección de residuos siempre ha estado asignado a la partida de servicios públicos municipales, de ahí que el porcentaje que recibe sea reducido, además de no contar con la posibilidad de cobrar por la prestación del servicio de manera autónoma. En este contexto, de acuerdo a la Dirección de Servicios Públicos del Municipio de Mexicali, el presupuesto anual que se destinó por parte del Ayuntamiento para la gestión de RSU en la ciudad de Mexicali y el Valle en 2012 fue de 148, 763,517 pesos mexicanos, equivalente al 6\% del total, porcentaje que el organismo municipal juzga insuficiente para alcanzar las metas del Plan de Desarrollo Municipal en el tema.

Educación y Capacitación. Desde el enfoque integral, se destacan los procesos de educación ambiental, formal y no formal y se incorpora de manera central el conocimiento del manejo jerárquico de los residuos, para potencializar la recuperación de materiales, y los términos de la capacitación para promotores, académicos, investigadores y servidores públicos en el tema. Desde este marco, el Ayuntamiento no ha implementado ejes de trabajo para el conocimiento de los RSU, solo la Dirección de Ecología Municipal ha elaborado folletos con temas ambientales en general. No se ha abordado la capacitación de los servidores públicos que se relacionan directa o indirectamente con la gestión de los residuos sólidos urbanos.

Investigación y Desarrollo. De acuerdo al enfoque de gestión integral para el manejo de RSU, se establece la necesidad de fortalecer la investigación y desarrollo científico, así como la innovación tecnológica, para 
reducir la generación de residuos y diseñar alternativas para su tratamiento, orientadas a procesos productivos más limpios, y establecer medidas de control. El municipio de Mexicali presenta escasas acciones de vinculación con instituciones académicas locales y regionales, que pudieran desarrollar propuestas de investigación e innovación tecnológica que contribuyan con el manejo de los RSU. La propuesta de mejoras tecnológicas ha estado sujeta a los términos de colaboración con la empresa concesionaria, que ha estado a cargo de la infraestructura para la transferencia y la disposición final.

Mercados. Con el enfoque de gestión integral se establece el fomento de la valorización de residuos, así como el desarrollo de mercados de subproductos, bajo criterios de eficiencia ambiental, tecnológicos y económicos con esquemas de financiamiento adecuados. A este respecto, En Mexicali, el mercado se mantiene informal y sin registro, preponderando actividades de la Unión de Bote y Chatarra, A.C., que ha signado compromisos con el Ayuntamiento y la empresa concesionaria.

Sistemas de Información. La LGPGIR (2003), establece que las autoridades de los tres órdenes de gobierno, en el ámbito de sus respectivas competencias, deben integrar un Sistema de Información sobre la Gestión Integral de Residuos. El municipio de Mexicali, no cuenta con sistemas de información sobre el tema de RSU. Algunos datos estadísticos están incorporados en la publicación de los planes o informes del gobierno municipal. El municipio cuenta con una oficina de atención para la transparencia y solicitud de información, pero no se localiza ninguna base de datos sobre medio ambiente y de manera específica sobre el tema de RSU.

Acceso a la información y comunicación. La LGPGIR (2003) establece el Derecho a la Información, en apego a la Ley de Transparencia y de Acceso a la Información Pública. En este aspecto, desde el quehacer del Ayuntamiento en materia de RSU, se informó que algunas acciones se comunican a través de la Dirección de Comunicación Social, como son los operativos de limpieza y campañas de eliminación de muebles viejos.

Seguimiento y Evaluación (monitoreo). El enfoque de gestión integral, establece que los tres órdenes de gobierno deben integrar sistemas de evaluación de las políticas de prevención de la contaminación. En el estudio de caso, no se cuenta con un proceso de seguimiento y evaluación de políticas para el manejo de RSU y de prevención de la contaminación.

\section{CONLUSIONES}

Como balance final se tiene que el Ayuntamiento de Mexicali se encuentra trabajando fundamentalmente bajo el enfoque básico, ya que no ha logrado incorporar y ejecutar los cambios propuestos en la ley de 2003 que lo pudieran acercan más al modelo de gestión sustentable, donde los aspectos de planeación se limitan a la programación anual de acciones. En este sentido la planeación sustentable de los RSU requiere de una visión estratégica de largo plazo, que actualmente no puede tener el municipio por ser administraciones trianuales, básicamente tácticas de pronta respuesta, pero que demandarían de un plan estratégico de largo plazo.

Instrumentar el enfoque de gestión sustentable de los residuos sólidos demanda cambios sustantivos de carácter estructural en lo normativo, ya que tendrían que ampliarse responsabilidades al municipio más allá de la gestión operativa que a la fecha tiene en la prestación del servicio, para así contar con un área de planeación que de seguimiento a través de las administraciones a programas y proyectos, realice su evaluación y mantenga la comunicación con la sociedad para retroalimentar la gestión.

La administración municipal puede moverse hacia la sustentabilidad si refuerza sus lazos con los actores externos: empresarios, academia y organizaciones de la sociedad civil que colaboren junto con las autoridades en la gestión RSU para diseñar una estrategia financiera, desarrollar investigación y tecnología apropiada, general información confiable, desarrollar indicadores para el monitoreo, capacitar y profesionalizar al personal y reforzar los programas de educación ambiental.

En la parte económica existe gran debilidad en la promoción de circuitos de Mercados y la formación de clústeres de empresas asociadas por parte de las autoridades, iniciativa privada y organizaciones de la sociedad civil. En este sentido a nivel municipal no se han planteado estrategias que involucren a los diferentes actores, ya que regularmente la actuación de los empresarios o comerciantes es aislada en temas como la reutilización y reciclado de autos, metales, materiales de construcción, papel, plásticos, muebles, electrónicos y ropa de segunda por citar algunos ejemplos.

Entre las bondades que tiene el enfoque de planeación y gestión sustentable de los RSU, es la apertura y valoración del trabajo interdisciplinario y transdisciplinario, donde concurren actores con diferentes capacidades en términos de capital humano y social, grupos que pueden incidir de manera positiva en la 
gestión del territorio, pero que requieren identificarse, organizarse, capacitarse, además de valorar sus recursos y niveles de interacción para que las acciones que desarrollen, tengan mayor impacto y visibilidad en la construcción de una ciudad o municipio saludable, éste representa uno de los retos que oriente el trabajo futuro de investigación para el caso de Mexicali.

Por último, los enfoques que abordan lo ambiental, el desarrollo y calidad de vida, subrayan el papel que desempeñan los actores en el proceso de planeación y gestión como parte del funcionamiento actual del modelo económico neoliberal, ya que anteriormente la gente desempeñaba un papel pasivo en el proceso de planeación y gestión municipal, para ahora convertirse en pieza clave del cambio. Sin embargo en la práctica, la gente todavía piensa que la gestión es solamente campo de las autoridades, de ahí que exista apatía en la participación y compromiso con proyectos que se promueven desde la autoridad. En este caso, se han estado haciendo esfuerzos a través de la educación formal y no formal para incidir en la cultura de la población, ver la oportunidad económica que representa la reutilización y reciclaje de residuos, así como la reducción en el consumo de recursos y uso eficiente de los mismos, cambios en estilos de vida, la importancia de la participación y el trabajo conjunto entre sociedad, autoridades y empresarios en la construcción de la agenda pública. Cambios que de lograrse superarían con creces el nivel que actualmente se tiene de participación informativa, consultiva y de validación de decisiones en el proceso de planeación, para transitar hacia la gobernanza.

\section{REFERENCIAS}

Alberta Urban Municipalities Association AUMA, Comprehensive guide for municipal sustainability planning (2006),

http://www.fraserbasin.bc.ca/_Library/SPC_Documents/1_1_c_AUMA_MSP_CompleteGuidebook_June06.p df. Acceso: 20 de Septiembre (2013).

Aponte, F. A., La sustentabilidad urbana en las ciudades, Boletim Goiano de Geografía Goiânia: 27 (2), 11 33 (2007).

Armijo, C., S. Ojeda-Benítez, E. Ramírez-Barreto y A. Quintanilla-Montoya, Potencial de reciclaje de los residuos de una institución de educación superior: el caso de la Universidad Autónoma de Baja California, Ingeniería: 10(3), 13-21 (2006).

Asociación Interamericana de Ingeniería Sanitaria y Ambiental - AIDIS, Directrices para la Gestión Integrada y Sostenible de Residuos Sólidos Urbanos en América Latina y el Caribe/Asociación Interamericana de Ingeniería Sanitaria y Ambiental - AIDIS y Centro Internacional de Investigaciones para el Desarrollo - IDRC, São Paulo (2006), http://www.aidis.org.br/PDF/libro_residuos_solidos.pdf Acceso: 20 de Septiembre (2013).

XVIII Ayuntamiento de Mexicali, Programa de Desarrollo Urbano de Centro de Población de Mexicali 2025, Periódico Oficial (2007).

Camacho-Gaxiola, E, Caracterización y comparación de los patrones de consumo en Mexicali, B.C., Tesis Maestría, Facultad de Arquitectura, Universidad Autónoma de Baja California, Mexicali, México (1995).

Centro Complutense de Estudios e Información Medioambiental, Programa Cambio Global España 2020/50 Programa Ciudades (2009), http://es.scribd.com/doc/32200399/CAMBIO-GLOBAL-ESPAN\%CC\%83A-202050. Acceso: 20 de Septiembre (2013).

Chen, X., Y. Geng, T. Fujita, An overview of municipal solid waste management in China, Waste Management: 30, 716-724 (2010).

Consorcio Cívico, Plan para la gestión sostenible de los residuos de origen domiciliario en Asturias: una alternativa a la incineración 2010 - 2040,

https://www.ecologistasenaccion.org/IMG/pdf_PLAN_SOSTENIBLE_RESIDUOS_AST.pdf. Acceso: 20 de Septiembre (2013).

Cortinas, C. y Y. Ordaz (Coord.), Informe de la situación general en materia de equilibrio ecológico y protección al ambiente 1993-1994, INE-SEMARNAT, México, D.F. (1994).

http://www2.inecc.gob.mx/publicaciones/libros/16/parte4_18.html. Acceso: 20 de Septiembre (2013).

CYMA, Guía informativa para la Elaboración de Planes Municipales de Gestión Integral de Residuos Sólidos (PMGIRS) $1^{\text {a }}$ edición, Programa Competitividad y Medio Ambiente (CYMA) en coordinación con la Asociación Centroamericana para la Economía, la Salud y el Ambiente (ACEPESA), San José, Costa Rica 
(2008). http://www.digeca.go.cr/documentos/residuos\%20solidos/2-Guia-Informativa-elaboracion-PlanesMunicipales-GIRS-2008.pdf. Acceso: 20 de Septiembre (2013).

Dálisa, G., M.F. Di Nola, M. Giampietro, A multi-scale analysis of urban waste metabolism: density of waste disposed in Campania, Journal of Cleaner Production: 35, 59-70 (2012).

De Valle, G, La Gestión Integral Sustentable de residuos sólidos urbanos: Diagnóstico y evaluación para la ciudad de Saltillo, Coahuila. El Colegio de México, México, D.F. (2005).

Diario Oficial de la Federación (DOF), Ley General del Equilibrio Ecológico y Protección al Ambiente (1988). http://www.oas.org/dsd/fida/laws/legislation/mexico/mexico_1988.pdf. Acceso: 20 de Septiembre (2013).

Diario Oficial de la Federación (DOF) Ley General para la Prevención y Gestión Integral de los Residuos (2003). http://www.diputados.gob.mx/LeyesBiblio/pdf/263.pdf. Acceso: 20 de Septiembre (2013).

Ezeah, C., C.L. Roberts, Waste governance agenda in Nigeria cities: a comparative analysis, Habitat International: 41, 121-128 (2014).

Favela, H, Cuantificación de residuos sólidos domésticos peligrosos generados en dos periodos estacionales en una ciudad mexicana, Memorias del II Simposio lberoamericano de Ingeniería de Residuos1-12, Barranquilla, Colombia 24 y 25 de Septiembre (2009).

Fierro, A, Análisis de la generación de residuos sólidos en supermercados de la ciudad de Mexicali, Revista Internacional de Contaminación Ambiental: 26 (4), 291-297 (2010).

Garduño, K., S. Ojeda, C. Armijo, Caracterización de residuos sólidos generados por el sector comercial de Mexicali, B.C., Revista Internacional de Contaminación Ambiental: 28(1), 19-25 (2012).

Gil, M. Á, Crónica del Instituto Nacional de Ecología, SEMARNAT. INE-SEMARNAT, México, D.F., (2009) http://www2.inecc.gob.mx/publicaciones/consultaPublicacion.html?id_pub=614. Acceso: 20 de Septiembre (2013).

Grupo Técnico del Inventario Mexicali, Inventario de emisiones de Mexicali (1999), Preparado por Ingeniería en Control Ambiental y Riesgo Industrial, S. de R. L. M. I., http://www.epa.gov/ttn/catc/dir1/mexicali.pdf. Acceso: 20 de Septiembre (2013).

Gutiérrez, V., Diagnóstico Básico para la gestión integral de residuos, SEMARNAT-INE, México, D.F., (2006), http://www2.inecc.gob.mx/publicaciones/download/495.pdf. Acceso: 20 de Septiembre (2013).

GTZ, La Basura en el Limbo: Desempeño de Gobiernos Locales y Participación Privada en el Manejo de Residuos Urbanos, México, D.F., Comisión Mexicana de Infraestructura Ambiental/ Agencia de Cooperación Técnica Alemana (GTZ) (2003), http://www2.gtz.de/dokumente/bib/07-0126.pdf. Acceso: 20 de Septiembre 2013.

ICLEI, Waste Solutions Six new approaches to sustainable urban waste management in Europe: Results of the European Urban Waste Management Cluster Projects. Dogliani, Italy, Local Governments for Sustainability, European Secretariat / European Commission (2005),

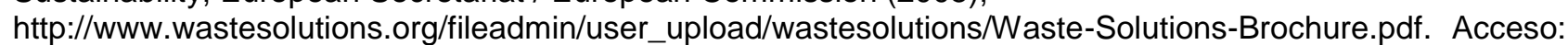
20 de Septiembre 2013.

INE, El manejo de los residuos sólidos urbanos y de manejo especial en México, México. D.F., Instituto Nacional de Ecología (2005), http://www2.inecc.gob.mx/publicaciones/libros/495/residuos.html. Acceso: 20 de Septiembre 2013.

INE, Generación y composición de los residuos sólidos municipales, En Reporte del estado ambiental y de los recursos naturales en la frontera norte de México por SEMARNAT, pp 191-199, México, D.F., (2010), http://www2.inecc.gob.mx/publicaciones/download/109.pdf. Acceso: 20 de Septiembre 2013.

INEGI, Residuos: Recolección y disposición final de residuos sólidos urbanos, 1998 a 2010 (2010), http://app1.semarnat.gob.mx/dgeia/informe_04/pdf/cap8.pdf. Acceso: 20 de Septiembre 2013. 
Jordán, R. y Simioni, D., Gestión Urbana para el Desarrollo Sostenible en América Latina y el Caribe, CEPAL, Santiago de Chile (2003), http://www.eclac.cl/publicaciones/xml/8/14288/lcg2203p.pdf. Acceso: 20 de Septiembre 2013.

Kanat, G., Municipal solid-waste management in Istanbul, Waste Management: 30, 1737-1745 (2010).

Kharvel, R., Sustainable Solid Waste Management in India, Master thesis, Department of Earth and Environmental Engineering, Columbia University, New York, U.S.A . (2012),

http://www.seas.columbia.edu/earth/wtert/sofos/Sustainable\%20Solid\%20Waste\%20Management\%20in\%20 India_Final.pdf. Acceso: 20 de Septiembre 2013.

Lohri, C.R., E.J. Camenzind, C. Zurbrügg, Financial sustainability in municipal solid waste management Costs and revenues in Bahir Dar, Ethiopia, Waste Management: 34(2), 542-552 (2014).

Marshall, R.E., K. Farahbakhsh, Systems approaches to integrated solid waste management in developing countries, Waste Management: 33, 988-1003 (2013).

Menikpura, S.N.M., J. Sang-Arun, M. Bengtsson, Integrated solid waste management: an approach for enhancing climate co-benefits through resource recovery, Journal of Cleaner Production: 58, 34-42 (2013).

Morinville, Morinville Municipal Sustainability Plan (2011). Alberta. Canada.

http://www.morinville.ca/index.php/strategic-business-plans/278-morinville-sustainability-plan. Acceso: 20 de Septiembre 2013.

Ojeda, S., R. Muñoz y F. González, Análisis estadístico del comportamiento de los residuos sólidos domiciliarios en una comunidad urbana, Frontera Norte: 10 (19), 65-76 (1998).

Ojeda, S. y H. Silva, El impacto de la contaminación por basura y sus efectos en la ciudad de Mexicali, B. C. (1996). Documento de trabajo, Instituto de Ingeniería, Universidad Autónoma de Baja California.

Okot-Okumu, J., R. Nyenje, Municipal solid waste management under descentrlisation in Uganda, Habitat International: 35, 537-543 (2011).

Papachristou, E., H. Hadjianghelou, E. Darakas, K. Alivanis, A. Belou, D. loannidou, E. Paraskevopoulou, K. Poulios, A. Koukourikou, K. Sortikos, Perspectives for integrated municipal solid waste management in Thessaloniki, Greece, Waste Management: 29, 1158-1162 (2009).

Pires, Ana, G. Martinho, N. Chang, Solid waste management in European countries: A review of systems analysis techniques, Journal of Environmental Management: 92, 1033-1050 (2011).

Seadon, J.K., Sustainable waste management systems, Journal of Cleaner Production: 18, 1639-1651 (2010).

SEDESOL, Estado de las Ciudades de México 2011, México, D.F., Secretaria de Desarrollo SocialPrograma de las Naciones Unidas para los Asentamientos Humanos, ONU-HABITAT (2011), http://www.onuhabitat.org/index.php?option=com_docman\&task=doc_details\&gid=583\&ltemid=330. Acceso: 20 de Septiembre 2013.

SEMARNAT, Programa Nacional para la Prevención y Gestión Integral de los Residuos 2009-2012, México, D.F., Secretaria de Medio Ambiente y Recursos Naturales (2009),

http://www.semarnat.gob.mx/informacionambiental/publicaciones/Publicaciones/SEMARNAT\%20Resumen \%20Ejecutivo\%2009.pdf. Acceso: 20 de Septiembre 2013.

SPA, Agenda Ambiental Metropolitana de Mexicali, Mexicali, México, Secretaría de Protección al Ambiente de Gobierno del Estado de Baja California (2011).

UNEP, Vital waste graphics Basel Action Network, Nairobi, Kenya, United Nations Environment Programme (2004). http://www.grida.no/publications/vg/waste/. Acceso: 20 de Septiembre 2013.

UNEP, Developing integrated solid waste management plan training manual. Volume 4: ISWM Plan, Osaka/Shiga, Japan, United Nations International Environmental Technology Centre (2009). http://www.unep.or.jp/ietc/publications/spc/iswmplan_vol4.pdf. Acceso: 20 de Septiembre 2013. 
UNEP, Emerging issues in our global environment (en línea), Nairobi, Kenya, United Nations Environment Programme (2012). http://www.unep.org/yearbook/2012/pdfs/UYB_2012_FULLREPORT.pdf. Acceso: 20 de Septiembre 2013.

Vij, D., Urbanization and solid waste management in India: present practices and future challenges, Procedia Social and Behavioral Sciences: 37, 437-447 (2012).

Zaman, A.U., Measuring waste management performance using "Zero Waste Index": the case of Adelaide, Australia, Journal of Cleaner Production: 66, 407-419 (2014).

Zaman, A.U., S. Lehmann, Urban growth and waste management optimization towards 'zero waste city', City, Culture and Society: 2, 177-187 (2011).

Zamorano, M., E. Molero, A. Grindlay, M.I. Rodríguez, A. Huertado, F. J. Calvo, A planning scenario for the application of geographical information systems in municipal waste collection; $A$ case of Churriana de la Vega (Granada, Spain), Resources, Conservation and Recycling: 54, 123-133 (2009). 
